\title{
THE VOLUME OF THE CEREBELLUM IN THE SECOND SEMESTER OF GESTATION
}

\author{
DAMIANA VULTURAR ${ }^{1}$, ALEXANDRU FĂRCĂȘANU ${ }^{2}$, FLAVIU TURCU ${ }^{2}$, \\ DAN BOITOR ${ }^{1}$, CARMEN CRIVII ${ }^{1}$
}

\author{
${ }^{1}$ Morphology Department, Iuliu Hatieganu University of Medicine and Pharmacy, \\ Cluj-Napoca, Romania \\ ${ }^{2}$ Physics Department, Babes-Bolyai University, Cluj-Napoca, Romania
}

\begin{abstract}
Background and aims. The cerebellum ("little brain"), the largest part of hind brain, lies in the posterior cranial fossa, beneath the occipital lobe and dorsal to the brainstem. It develops over a long period: it is one of the first structures in the brain to begin to differentiate, but one of the last to mature. The use of ultrasonography has significantly improved the evaluation of fetal growth and development and has permitted prenatal diagnosis of a variety of congenital malformations.

The aim of our study was to evaluate the cerebellar growth and development using 2 different measuring techniques: microMRI and ultrasound technique. The cerebellum measurements were related to gestational age.

Methods. We used 14 human fetuses corresponding to 15-28 gestational weeks, immersed in a 9\% formalin solution. Magnetic Resonance Imaging (MRI) was performed byemploying a Bruker BioSpec 70/16USR scanner (Bruker BioSpin MRI GmbH, Ettlingen, Germany), operated at 7.04 Tesla for cerebellar volume measurement. Ultrasonographic measurements of the cerebellum diameter were performed on 14 pregnant women, 15 - 28 gestational weeks. Ultrasound scan used 5-10 MHZ for transvaginal approach. Taking into consideration the values of the cerebellum dimensions and considering the general shape of the cerebellum as a transverse ellipsoid, the volume of the cerebellum was calculated by a mathematical formula for ellipsoid volume.

Results. The study correlates the measurements from the microMRI study with the ultrasounds data and the results are superposable. Both established the exponential volume growth after the 22-23 GW. We used the ellipsoid volume formula for the cerebellar volume using the half of the three diameters of the cerebellum determined by ultrasound measurements:

Cerebellar Volume $=$ Ellipsoid volume $=3 / 4 \pi r 1$ r2 $r 3$

Conclusion. There is a linear correlation between the microMRI measurements and ultrasound determinations. Based on all collected data we could apply an easy formula to calculate the volume of cerebellum, a useful criterion in the evaluation of the cerebellar development and the appreciation of the gestational age.
\end{abstract}

Keywords: cerebellum development, cerebellum fetal dimensions, cerebellar volume

\section{Background and aims}

The normal evolution of fetal development is an important aspect in obstetric practice and the correct appreciation of the transformative parameters is continually changing owing to the progress of technical investigations.

Manuscript received: 10.07.2017

Received in revised form: 25.11.2017

Accepted: 06.12.2017

Address for correspondence: bianca.crivii@umfcluj.ro
The ultrasonographic and magnetic resonance investigations give us the possibility to appreciate more accurately the parameters of the fetal evolution related to the gestational age. Recently, the physiological development of the brain is appreciated based on the dimensions of the skull or of the brain. Along with Schmahmann's explanations regarding the cognitive affective functions of the cerebellum [1], the cerebellum has become a common topic of study and 
evaluation of fetal development.

The cerebellum ("the little brain"), located in the posterior cranial fossa, beneath the occipital lobe and dorsal to the brainstem, exposes the vermis - a midline region, a narrow adjacent area (paravermian area) and the hemispheres on each side. The cerebellum is divided by the fissures in flocculonodular, anterior and posterior lobes. Except the first one, the lobes are divided further in lobules and laminae (folia) to increase the cerebellar surface. The cerebellum has a very well organized architecture with a major role in sensory-motor control: integrating the inputs from the sensory system or other parts of the brain, it contributes to motor coordination, precision, accurate timing and motor learning. In addition, the cerebellum is involved in cognitive functions (attention, language) and in emotion (the response to pleasure and fear). The cerebellar dysfunctions consist in disorders of equilibrium, posture, automatic/fine movements and motor learning. The cerebellum development lengthens from the early embryonic time until the postnatal years. Therefore the development of cerebellum presents a high susceptibility in undergoing a large spectrum of developmental disorders such as malformations and disruptions.

As a part of the encephalon, the cerebellum development starts with the neurulation - the folding process of the neural plate from the ectodermal layer. The neural plate turns into neural tube with a rostral part, base for consecutive divisions, developing the brain vesicles: the forebrain (prosencephalon) - which gives the telencephalon and diencephalon, the midbrain (mesencephalon) and the hindbrain (rhombencephalon) which gives metencephalon and myelencephalon.

The cerebellum develops from the alar plate, the dorsal part of the metencephalon, and the neural folds - the future rhombic lips. The alar plate expands laterally and form the rhombomeres 1. By medial fusion they delimit the cavity of the IVth ventricle and a smooth - convex structure, the rudiment of the cerebellum with the midline vermis. The cerebellar fissures are seen starting with 3-rd month of developing, on the vermis surface and floccular region, and from 5th month to the level of the hemispheres [2-4]. The mass of cerebellum will double after the 19th week of gestation and will grow even after birth [5-8].

Clinically, the evolution of cerebellum gives information related to the developmental stage of the fetus. Using the ultrasonographic technique or MRI investigation, recent studies determine different parameters of cerebellar development to establish the developmental age. Ultrasound studies highlight the importance of the transverse cerebellar diameter (TCD) or vermis dimensions as an alternative procedure to the appreciation of gestational age by the measurement of biparietal diameter (BPD) of the skull [9-12]. Another tool of imaging determination, the MRI technique, gives the possibility to study the volume of developmental cerebellum as a more accurate parameter in relation with gestational age.

\section{Methods}

The microMRI study used 14 human fetuses corresponding to 15-28 gestational weeks from the Collection of the Anatomy - Embryology Department. They were preserved in a $9 \%$ formalin solution during the first 48 hours at $4^{\circ} \mathrm{C}$, and were subsequently stored at room temperature. They had normal external morphology. Magnetic Resonance Imaging (MRI) was performed by using a Bruker BioSpec 70/16USR scanner (Bruker BioSpin MRI GmbH, Ettlingen, Germany), operated at 7.04 Tesla. All the experiments were performed at room temperature $\left(20^{\circ} \mathrm{C}\right)$ with fetuses immersed in a $4 \%$ formaldehyde solution inside plastic cylindrical containers. The adjustments of the anatomical references were obtained by $\mathrm{T} 2$-weighted images (T2-wi), acquired in all three orthogonal planes: axial, coronal and sagittal planes.

For 3D reconstruction of the developing human nervous system the Fast Imaging with Steady-state Precession (FISP) sequence was engaged. Following the acquisition and processing of 2D slices with Bruker's ParaVision ${ }^{\circledR}$, the $3 \mathrm{D}$ reconstruction of the investigated embryos was performed using the InVesalius software (Figure 1).
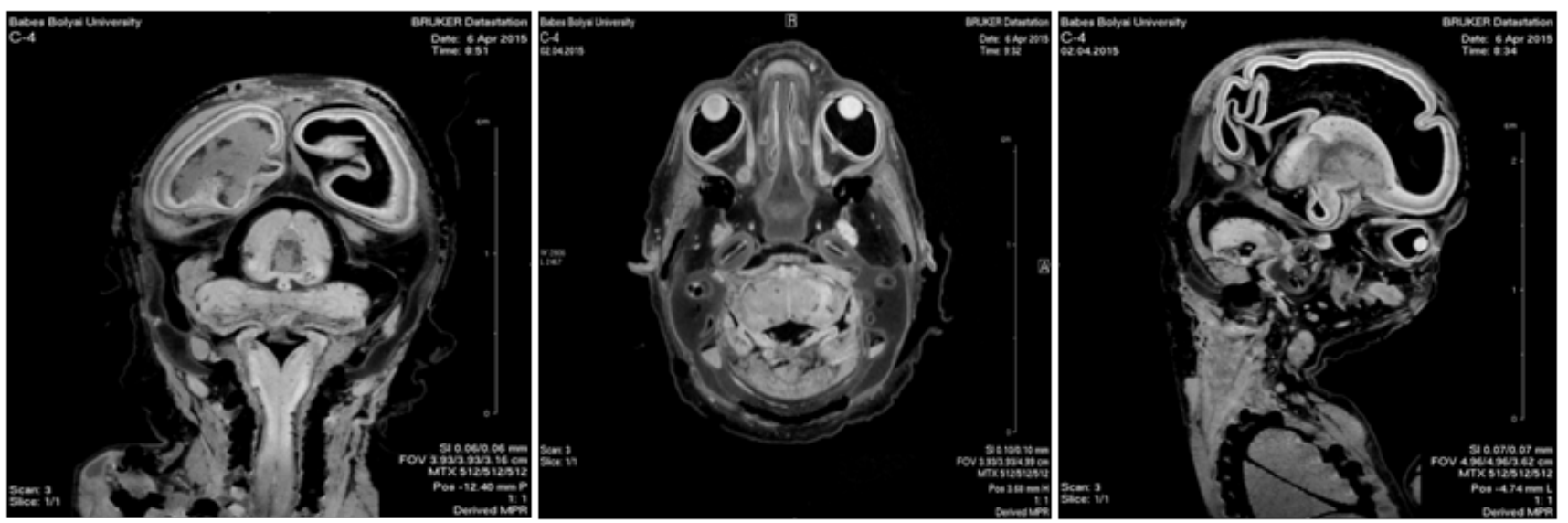

Figure 1. 15 GW fetus - microMRI images of the axial, coronal and parasagittal planes. 
Ultrasound scan was performed in 14 pregnant women with physiological evolution, 15 - 28 gestational weeks. Exclusion criteria were multiple gestation pregnancy, brain abnormalities. Mothers provided informed consent for using fetal scans for research purposes. Ultrasound scan used 5-10 MHZ by transvaginal approach. The cerebellum can be visualized as a "butterfly" shaped structure with two hemispheres that are joined in the middle by the vermis - more echogenic than the hemispheres. The images were taken by $3 \mathrm{D}$ transvaginal probe to examine the fetus development and we measured the three diameters of cerebellum: transverse cerebellar diameter (TCD), depth and height of cerebellar lobes (Figure 2).

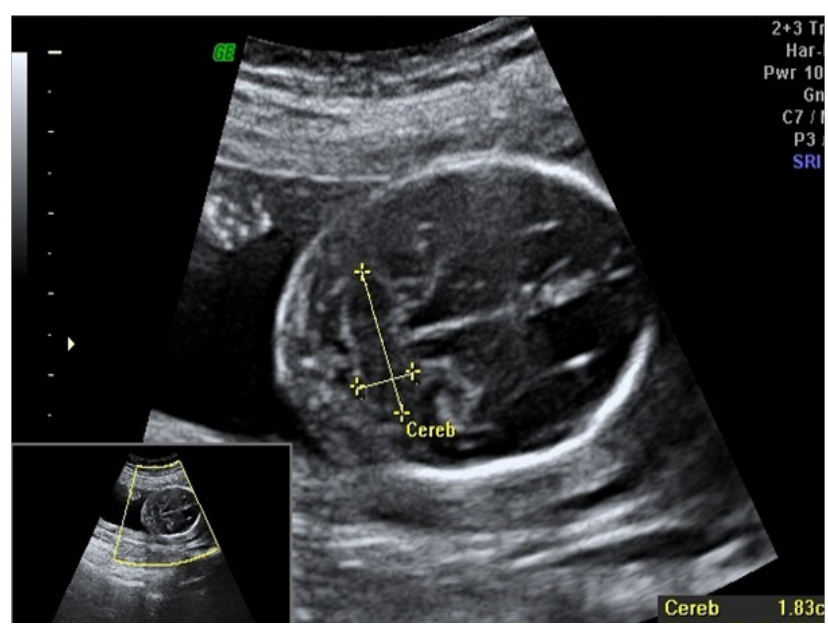

Figure 2. $17 \mathrm{GW}$ fetus - ultrasound image - transverse section of the head of the fetus.

Because of the similarity between cerebellum shape and an ellipsoid, we used the mathematic formula for the volume calculation based on the ultrasonographic measurements taking in consideration the three diameters: ellipsoid volume $=\mathbf{3} / \mathbf{4} \boldsymbol{\pi} \mathbf{r} 1 \mathbf{r} 2 \mathbf{r}$, where $r 1, r 2, r 3$ are the half diameters of the three dimensions. The calculation was made automatically with the calculator from a web-page [13].

Ethical approval - the study protocol was approved by the Ethics Committee of the Iuliu Hatieganu Medicine and Pharmacy University of Cluj, Romania.

\section{Results}

Cerebellar volumes from the microMRI study are synthesized in table I.

Data from ultrasound scan determinations of cerebellum dimensions and for the calculating volume according to the ultrasounds measurements are synthesized in table II.
Table I. The values of the cerebellar volume determinated by microMRI study.

\begin{tabular}{ll}
\hline $\mathrm{GW}$ & Volume $\left(\mathrm{mm}^{3}\right)$ \\
\hline 15 & 498 \\
\hline 16 & 656 \\
\hline 17 & 802 \\
\hline 18 & 986 \\
\hline 19 & 1198 \\
\hline 20 & 1358 \\
\hline 21 & 1596 \\
\hline 23 & 2028 \\
\hline 24 & 2466 \\
\hline 25 & 2890 \\
\hline 26 & 3418 \\
\hline 27 & 3880 \\
\hline 28 & 4558 \\
\hline
\end{tabular}

Table II. Values of the cerebellum ultrasounds measurements and the calculating volumes.

\begin{tabular}{lllll}
\hline GW & $\begin{array}{l}\text { Width } \\
(\mathrm{mm})\end{array}$ & $\begin{array}{l}\text { Depth } \\
(\mathrm{mm})\end{array}$ & $\begin{array}{l}\text { Height } \\
(\mathrm{mm})\end{array}$ & $\begin{array}{l}\text { Ellipsoid Volume } \\
\left(\mathrm{mm}^{3}\right)\end{array}$ \\
\hline 15 & 16.4 & 7.2 & 8.4 & 505.7102 \\
\hline 16 & 17.2 & 7.6 & 9.6 & 655.4277 \\
\hline 17 & 18.3 & 8.2 & 10.4 & 815.0979 \\
\hline 18 & 18.9 & 9 & 11.4 & 1012.793 \\
\hline 19 & 20.3 & 9.8 & 12.2 & 1267.633 \\
\hline 20 & 20.8 & 9.9 & 12.8 & 1376.639 \\
\hline 21 & 22.7 & 10.8 & 13.2 & 1690.188 \\
\hline 22 & 23.9 & 11.9 & 13.8 & 2049.913 \\
\hline 23 & 24.6 & 12.6 & 15.9 & 2574.034 \\
\hline 24 & 26.8 & 13 & 17.2 & 2893.256 \\
\hline 25 & 28.3 & 13.4 & 18.1 & 3584.935 \\
\hline 26 & 29.4 & 14.2 & 17.8 & 3881.211 \\
\hline 27 & 30.4 & 16.1 & 18.2 & 4652.457 \\
\hline 28 & 31.6 & 16.2 & 18.4 & 5230.987 \\
\hline & & & &
\end{tabular}

\section{Discussion}

In the last decades the ultrasound investigation of the fetal evolution has become a clinical routine. An important number of papers offer data on the fetal development. The study of fetal cerebellum [14,15] represents an important subject in the attempt to find parameters of the physiological fetal evolution. There are papers sustaining the importance of the TCD [16] or the vermian dimensions as relevant parameters of the fetal evolution [15], but other papers [14,17] emphasized the importance of the global appreciation of the fetal cerebellum as is the cerebellar volume. Based on the MRI investigations $[17,18]$ the $3 \mathrm{D}$ determinations are more precise, more accurate, and the 
Cerebellar Volume (mm3)

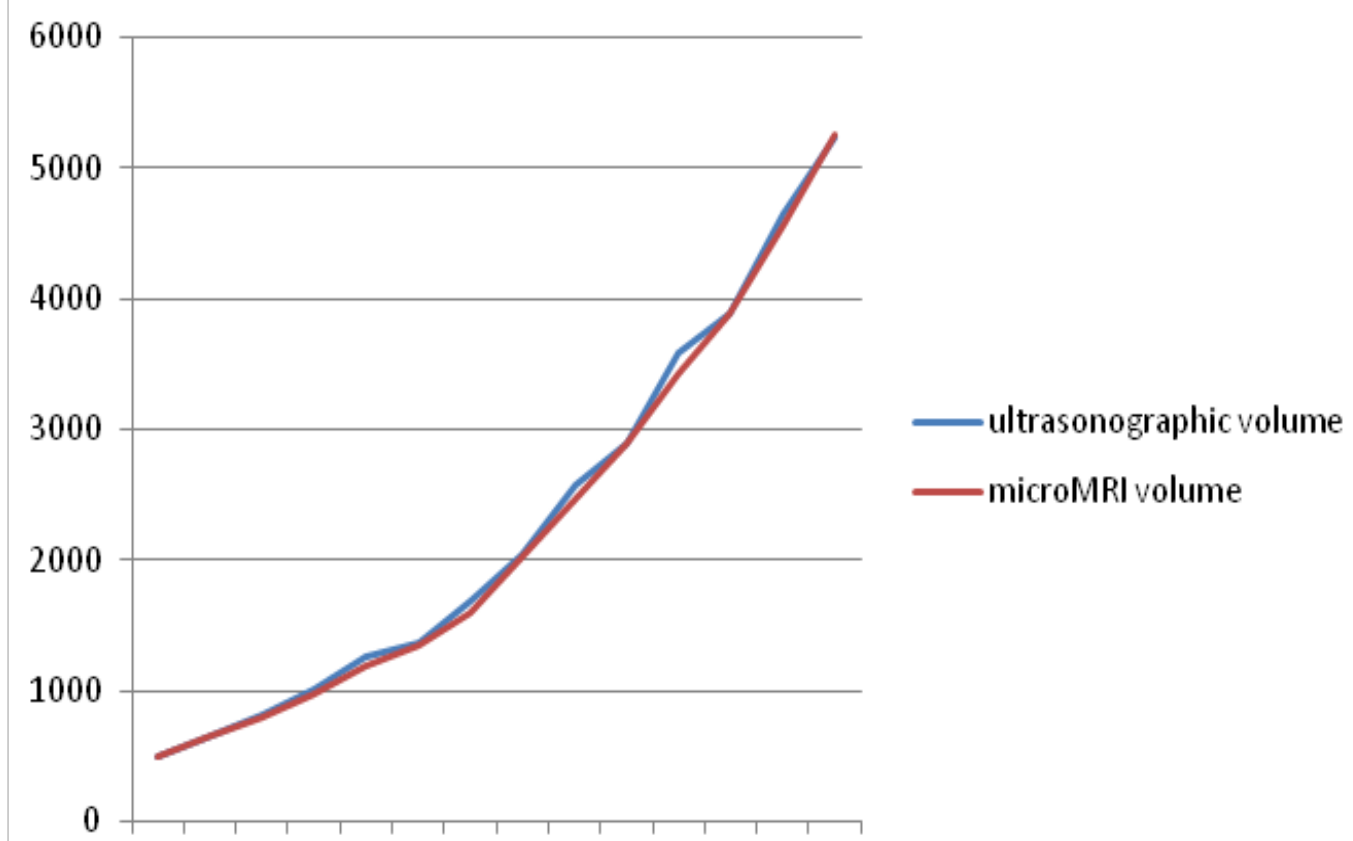

1516171819202122232425262728

Gestation weeks

Figure 3. The evolution of the fetal cerebellar volume determinations by the two techniques: microMRI and ultrasound.

information can be a reference frame for the cerebellum evolution and the evaluation of gestational week. But the MRI investigation is not a routine investigation, ultrasound determination being the current investigation of the fetal evolution. The ultrasound technique is an easy way to find different parameters of the fetal structures in evolution. For the cerebellum, the volume can be calculated based on the three diameters: TCD, width and depth of the cerebellar hemispheres. Cerebellum has a similar shape with a transverse ellipsoid with an easy formula for its volume. We used this formula to calculate the cerebellar volume and we compared the data with the results from microMRI determinations.

Regardless of whether the cerebellum is not a perfect ellipsoid, the evolution of the two different determinations are obviously superposable (Figure 3 ). The explanation lies in the fact that at these dimensions, the differences between the perfect ellipsoid and the cerebellum are negligible. Both established the exponential volume growth after the 22-23 GW.

Compared to other studies, the data related to the depth of cerebellum from our study are similar with the data from Cignini study [15]. As for the cerebellar volume, our results are higher than the results of Ber study [17] with $8.83 \%, 20.57 \%$, respectively $24.06 \%$ for the $26-28$ $\mathrm{GW}$, they are coming between $-3 \% /+13 \%$ compared to the results of Hatab volumetric study [18], are lower than the results of Zhan study [19], but similar with the results of Liu study [20].

Taking into consideration our study and the results from other studies, we conclude that for the evolution of fetal cerebellum the volume determination is an accurate parameter and it can be calculated applying the ellipsoid formula, the differences between real cerebellar volume and the ellipsoid volume with same diameters being negligible.

\section{Conclusions}

There is a linear correlation between the microMRI measurements and ultrasound determinations. On the basis of all collected data we could apply the transverse ellipsoid formula for cerebellar volume calculation, a useful criterion in evaluation of the cerebellum development and the gestational age. Studies of embryology are still needed for a complete assessment of the developing organs. This can provide a better understanding of the normal and pathological morphogenesis.

\section{References}

1. Schmahmann JD, Sherman JC. Cerebellar cognitive affective syndrome. Int Rev Neurobiol. 1997;41:433-440.

2. Marzban H, Del Bigio MR, Alizadeh J, Ghavami S, Zachariah RM, Rastegar M. Cellular commitment in the developing 
cerebellum. Front Cell Neurosci. 2015 Jan 24;8:450. doi: 10.3389/ fncel.2014.00450.

3. Ito M. Cerebellar circuitry as a neuronal machine. Prog Neurobiol. 2006;78(3-5):272-303.

4. Leto K, Arancillo M, Becker EB, Buffo A, Chiang C, Ding B, et al. Consensus Paper: Cerebellar Development. Cerebellum. 2016;15(6):789-828.

5. Guihard-Costa AM, Larroche JC. Differential growth between the fetal brain and its infratentorial part. Early Hum Dev. 1990;23(1):27-40.

6. Guihard-Costa AM, Larroche JC. Growth velocity of some fetal parameters. I. Brain weight and brain dimensions. Biol Neonate. 1992;62(5):309-316.

7. Co E, Raju TN, Aldana O. Cerebellar dimensions in assessment of gestational age in neonates. Radiology. 1991;181(2):581-585.

8. Triulzi F, Parazzini C, Righini A. MRI of fetal and neonatal cerebellar development. Semin Fetal Neonatal Med. 2005;10(5):411-420.

9. Araujo Júnior E, Martins WP, Nardozza LM, Pires CR, Filho SM. Reference range of fetal transverse cerebellar diameter between 18 and 24 weeks of pregnancy in a Brazilian population. J Child Neurol. 2015;30(2):250-253.

10. Leung TN, Pang MW, Daljit SS, Leung TY, Poon CF, Wong SM, et al. Fetal biometry in ethnic Chinese: biparietal diameter, head circumference, abdominal circumference and femur length. Ultrasound Obstet Gynecol. 2008;31:321-327.

11. Vinkesteijn AS, Mulder PG, Wladimiroff JW. Fetal transverse cerebellar diameter measurements in normal and reduced fetal growth. Ultrasound Obstet Gynecol. 2000;15(1):47-51.

12. Serhatlioglu S, Kocakoc E, Kiris A, Sapmaz E, Boztosun Y, Bozgeyik Z. Sonographic measurement of the fetal cerebellum, cisterna magna, and cavum septum pellucidum in normal fetuses in the second and third trimesters of pregnancy. J Clin Ultrasound. 2003;31(4):194-200.

13. http://www.csgnetwork.com/volumeellipsoid.html

14. Scott JA, Hamzelou KS, Rajagopalan V, Habas PA, Kim K, Barkovich AJ, et al. 3D morphometric analysis of human fetal cerebellar development. Cerebellum 2012;11(3):761-770.

15. Cignini P, Giorlandino M, Brutti P, Mangiafico L, Aloisi A, Giorlandino C. Reference Charts for Fetal Cerebellar Vermis Height: A Prospective Cross-Sectional Study of 10605 Fetuses. PLoS One. 2016 Jan 26;11(1):e 0147528. doi: 10.1371/journal. pone.0147528.

16. Naseem F, Fatima N, Yasmeen S, Saleem S. Comparison Between Transcerebellar Diameter with Biparietal Diameter of Ultrasound for Gestational Age Measurement in Third Trimester of Pregnancy. J Coll Physicians Surg Pak. 2013;23(5):322-325.

17. Ber R, Hoffman D, Hoffman C, Polat A, Derazne E, Mayer A, et al. Volume of Structures in the Fetal Brain Measured with a New Semiautomated Method. AJNR Am J Neuroradiol. 2017;38(11):2193-2198.

18. Hatab MR, Kamourieh SW, Twickler DM. MR volume of the fetal cerebellum in relation to growth. J Magn Reson Imaging. 2008;27:840-845.

19. Zhan J, Dinov ID, Li J, Zhang Z, Hobel S, Shi Y, et al. Spatialtemporal atlas of human fetal brain development during the early second trimester. Neuroimage. 2013;82:115-26.

20. Liu F, Zhang Z, Lin X, Teng G, Meng H, Yu T, et al. Development of the human fetal cerebellum in the second trimester: a post mortem magnetic resonance imaging evaluation. J Anat. 2011;219(5):582-588. 